\title{
EFFECT OF SMOKING ON THE CLINICAL AND LABORATORY PRESENTATION OF PATIENTS WITH SYSTEMIC SCLEROSIS IN A LARGE BRAZILIAN COHORT.
}

Osvaldo Luiz Camata Junior (Universidade de São Paulo, São Paulo, SP, Brasil), Rodrigo Vidaurre (Universidade de São Paulo, São Paulo, SP, Brasil), Adriana Bruscato Bortoluzzo (Instituto de Educação e Pesquisa, São Paulo, SP, Brasil), Ana Paula Luppino-Assad (Universidade de São Paulo, São Paulo, SP, Brasil), Leandro Lara do Prado (Universidade de São Paulo, São Paulo, SP, Brasil), Henrique Carriço da Silva (Universidade de São Paulo, São Paulo, SP, Brasil), Danieli Castro O. Andrade (Universidade de São Paulo, São Paulo, SP, Brasil), Percival D. Sampaio-Barros (Universidade de São Paulo, São Paulo, SP, Brasil)

\section{BACKGROUND}

Smoking has been recently identified as a risk factor for Raynaud phenomenon, digital ulcers and decrease in lung function in systemic sclerosis (SSc). However, many times we can have distinct and controversial results, thus underestimating the effect of smoking on SSc. The aim of this study is to identify the impact of smoking on the clinical and laboratory presentation of SSc, analyzing its influence on disease outcomes, compared to non-smoking SSc patients.

\section{MATERIALS AND METHODS}

This retrospective study analyzed 700 consecutive patients classified as SSc according to the 2013 ACR/ EULAR criteria attended in a single SSc outpatient clinic in the period from 2010 to 2018. Demographic, clinical and laboratory data, as well as smoking (package/yr, number of smoking years), pulmonary function test and chest high resolution computed tomographic (HRCT) data were obtained from an electronic register database. Statistical significance was considered if $p<0.05$.

\section{RESULTS}

Among the 700 SSc patients, 477 (68.1\%) were never smokers, 178 (25.4\%) ex-smokers and 45 (6.4\%) current smokers. When comparing smokers (current + ex) with non-smokers, those smokers presented higher age at onset $(45.53+12.40 y r s$ vs. $40.87+14.62 y r s ; p<0.001)$ and age at diagnosis $(47.63+12.45 y r s$ vs. $43.06+14.83 y r s ; p<0.001)$, and association with male gender $(p<0.001)$, sSc sine scleroderma $(p=0.018)$, exposition to environmental factors $(p=0.001)$, heart involvement $(p=0.019)$, congestive heart insufficiency $(p<0.001)$, and use of home $02(p=0.004)$; smoking also presented negative association with pigmentary disturbances $(p=0.035)$ and anti-Scl70 ( $p=0.034)$. Interestingly, when we compared exsmokers with current smokers we found that ex-smokers presented association with higher mean Rodnan skin score $(8.40+8.61$ vs. $5.22+4.57 ; p=0.001)$, non-diffuse SSc $(p=0.035)$, exposition to environmental factor $(p=0.008)$, worse dyspnea - NYHA class $(p=0.024)$, fibrosis on chest HRCT $(p=0.002)$, positive antiScl70 ( $p=0.036)$, use of cyclophosphamide $(p=0.010)$ and mycophenolate $(p=0.023)$; the mean number of package/years was $28.85+16.28$ for the current smokers and $22.10+15.33$ for the ex-smokers $(p=0.023)$. When comparing current smokers with non-smokers, current smokers were positively associated with male gender $(p=0.031)$, diffuse SSc $(p=0.047)$, congestive heart failure $(p=0.045)$ and Barrett esophagus $(p=0.037)$; conversely, they were negatively associated with mean Rodnan skin score $(5.22+4.57$ vs. $8.37+8.29 ; p<0.001)$, fibrosis at the chest HRCT $(p=0.019)$, positive anti-Scl70 $(p=0.006)$ and use of cyclophosphamide $(p=0.007)$ and mycophenolate $(p=0.017)$.

\section{CONCLUSION}


Despite being associated with lower anti-Scl70 positivity, smoking was clearly associated with worse prognosis in this cohort when considered smokers vs. non-smokers. The comparison between current vs ex-smokers showed that SSc patients with higher fibrotic skin and lung components, especially when antiScl70 positive, had a trend to quit smoking earlier. 